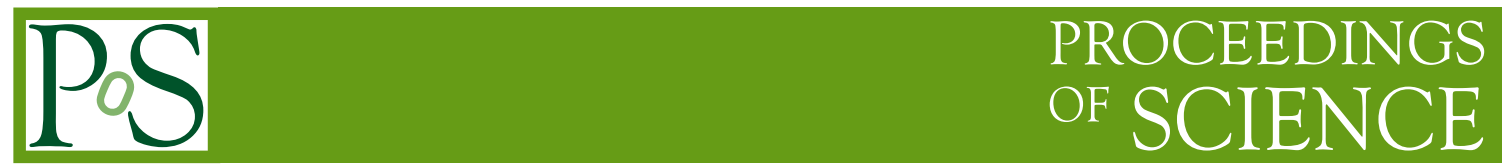

\title{
Flavor Physics in Beyond Standard Models
}

\author{
Yuji Omura* \\ Kobayashi-Maskawa Institute for the Origin of Particles and the Universe, \\ Nagoya University, Nagoya 464-8602, Japan \\ E-mail: yujiomur@kmi.nagoya-u.ac.jp
}

We discuss flavor physics to prove new physics beyond the Standard Model (SM). There are many candidates for extended Standard Models. First, we focus on the SO(10) Grand Unified Theory (GUT) that explains the origin of the SM gauge group, and propose flavor violating processes relevant to the GUT. As another candidate for new physics, we also discuss a simple extended SM to introduce dark matter, and explore the possibility that dark matter is tested by flavor physics.

The 3rd International Symposium on "Quest for the Origin of Particles and the Universe" 5-7 January 2017

Nagoya University, Japan

${ }^{*}$ Speaker. 
There are a lot of motivations to consider extensions of the Standard Model (SM). One of the mysteries to stimulate physicists to search for new physics is the non-trivial structure of the SM gauge symmetry. The SM is governed by $S U(3) \times S U(2)_{L} \times U(1)_{Y}$ gauge symmetry and the $S U(2)_{L} \times U(1)_{Y}$ charge assignment for quarks and leptons is very unique. The gauge anomaly-free conditions are miraculously satisfied in the SM, so that this structure seems to suggest new physics behind the SM to reveal the origin of this SM gauge symmetry.

One of the promising candidates for the origin is an $S O(10)$ supersymmetric Grand Unified theory (SUSY GUT). The gauge symmetries $S U(3) \times S U(2)_{L} \times U(1)_{Y}$ can be embedded into $\mathrm{SO}(10)$ gauge group introducing one extra $\mathrm{U}(1)$ symmetry, and we can understand the reason why the anomaly-free conditions are satisfied in the SM. In the minimal SO(10) GUT, the SM quarks and leptons are naturally embedded into three 16-dimensional fields [1]. Thus, the $S O(10)$ GUT has been studied for a long time as a promising candidate for new physics. This extension, however, suffers from the inconsistency with the experimental results of the fermion mass spectrum. This problem is caused by the matter unification mentioned above, so that we have to consider a nonminimal setup of $S O(10)$ GUT for realistic mass matrices of quarks and leptons. In Refs. [2, 3], the authors consider a simple setup motivated by this issue and discuss flavor physics to prove the GUT model. In the model, the SM quarks and leptons come from both 10- and 16-dimensional fields, and especially, the right-handed down-type quarks and left-handed leptons in the SM are given by the linear combinations of 10- and 16-dimensional fields. Then, the matter unification is spoiled at low energy and the realistic mass spectrum can be realized. The $S O(10)$ gauge symmetry breaks down to $S U(3)_{c} \times S U(2)_{L} \times U(1)_{Y} \times U(1)^{\prime}\left(\equiv G_{S M} \times U(1)^{\prime}\right)$ around $10^{16} \mathrm{GeV}$. Thus, the low-energy effective theory is an $U(1)^{\prime}$ extension of the SUSY SM with extra matters that are predicted by $\mathbf{1 0}$ - and 16-dimensional fields. The additional gauge symmetry will survive up to the SUSY scale, but we could expect that it is radiatively broken, as the electroweak (EW) symmetry breaking in the minimal supersymmetry Standard Model (MSSM). We assume that SUSY particles in the SUSY SM, except for gauginos, reside around $100 \mathrm{TeV}$, in order to realize the observed $125 \mathrm{GeV}$ Higgs mass and also to satisfy constraints on flavor- and/or CP-violating processes.

The mass of the $Z^{\prime}$ boson associated with the gauged $U(1)^{\prime}$ may be $O(100) \mathrm{TeV}$ corresponding to the SUSY scale, so that it may be viable in the searches for flavor violations. The right-handed down-type quarks and left-handed leptons in the SM are given by linear combinations of the parts of 10- and 16-dimensional fields. This generically leads to flavor-violating $Z^{\prime}$ interaction and crucial promises against flavor experiments. We will see that tree-level Flavor Changing Neutral Currents (FCNC) induced by the $Z^{\prime}$ boson are generated and they largely contribute to the flavor violation processes: for instance, $\mu \rightarrow 3 e, \mu-e$ conversion in nuclei, and $K^{0}-\bar{K}^{0}$ and $B_{d / s}^{0}-\bar{B}_{d / s}^{0}$ mixings. In particular, we find that the $\mu$-e conversion and the CP-violation in $K$ system provide the strongest constraints with our model.

In this paper, we also explore another possibility of extensions of the SM. The SO(10) GUT discussed above is a hypothesis that new physics reside at very high energy. We will suggest flavor physics to test such a high-scale physics, but it would be very difficult to prove the model explicitly and directly. As another way to find new physics, we take phenomenological approach. One big issue among the experimental results is the existence of dark matter (DM). The WMAP and the Planck experiments have shown that the relic density of DM is about 5 times larger than the one of the baryon in our universe $[4,5]$. Such a large DM density implies the existence of physics 
beyond the SM. A lot of ideas motivated by DM have been proposed so far. One simple idea is as follows. DM candidate is singlet under the SM gauge groups. Then, DM couples with the SM Higgs field and the SM fermions, introducing extra (colored) fields. This kind of DM model has been proposed, in the framework of the GUT [6], and it is one of possible and attractive setups. In Ref. [7], the authors have discussed not only DM physics but also flavor physics to prove the simplified DM model. It is interesting that the future experiment of the DM direct detection can prove this model and the measurement of $\Delta F=2$ processes with high accuracy can conclude this DM scenario.

In this paper, we discuss these two different approaches for new physics and propose various flavor violating processes relevant to each setup, following Refs. [2, 3, 7, 8]. We see that different processes become important depending on the motivations of new physics.

\section{Study of $S O(10)$ SUSY GUT}

First, we discuss the SO(10) GUT, based on Refs.[2, 3]. We introduce a 10-dimensional matter field in the each generation in addition to 16-dimensional matter fields. Three $S O(10)$-singlet matter fields $S_{i}$ are also introduced to achieve the realistic masses of neutrinos [2,3]. The matter fields $\mathbf{1 0}_{i}$ and $\mathbf{1 6}_{i}$ are decomposed as the ones in Table 1. For convenience, the assignment of $S U(5) \times U(1)^{\prime}$ is also shown in Table 1 . The superpotential is written down according to the

\begin{tabular}{|c|c|c|c|c|c|c|}
\hline & $Q_{L}$ & $U_{R}^{c}$ & $E_{R}^{c}$ & $\hat{L}_{L}$ & $\hat{D}_{R}^{c}$ & $N_{R}^{c}$ \\
\hline$S O(10)$ & \multicolumn{6}{|c|}{16} \\
\hline$S U(5) \times U(1)^{\prime}$ & \multicolumn{3}{|c|}{$(\mathbf{1 0},-1)$} & \multicolumn{2}{|c|}{$(\overline{\mathbf{5}}, 3)$} & $(1,-5)$ \\
\hline$G_{\mathrm{SM}}$ & $\left(\mathbf{3}, \mathbf{2}, \frac{1}{6}\right)$ & $\left(\overline{\mathbf{3}}, \mathbf{1},-\frac{2}{3}\right)$ & $(\mathbf{1}, \mathbf{1}, 1)$ & $\left(\mathbf{1}, \mathbf{2},-\frac{1}{2}\right)$ & $\left(\overline{\mathbf{3}}, \mathbf{1}, \frac{1}{3}\right)$ & $(\mathbf{1}, \mathbf{1}, 0)$ \\
\hline & $L_{L}^{\prime}$ & $\bar{C}^{\prime D_{R}^{\prime c}}$ & $\overline{\overline{L^{\prime}}}$ & $\overline{\bar{~} \overline{D_{R}^{\prime c}}}$ & & \\
\hline$S O(10)$ & \multicolumn{4}{|c|}{10} & & \\
\hline$S U(5) \times U(1)^{\prime}$ & \multicolumn{2}{|c|}{$(\overline{\mathbf{5}},-2)$} & \multicolumn{2}{|c|}{$(\mathbf{5}, 2)$} & & \\
\hline$G_{\mathrm{SM}}$ & $\left(\mathbf{1}, \mathbf{2},-\frac{1}{2}\right)$ & $\left(\overline{\mathbf{3}}, \mathbf{1}, \frac{1}{3}\right)$ & $\left(\mathbf{1}, \mathbf{2}, \frac{1}{2}\right)$ & $\left(\mathbf{3}, \mathbf{1},-\frac{1}{3}\right)$ & & \\
\hline
\end{tabular}

Table 1: Charge assignment for matter fields. Charge assignment for $G_{\mathrm{SM}}$ is denoted as $\left(S U(3)_{c}, S U(2)_{L}\right.$, $\left.U(1)_{Y}\right)$.

$S O(10)$ gauge symmetry, adding $\mathbf{1 0}_{H}, \mathbf{1 6}_{H}$ and $\overline{\mathbf{1 6}}_{H}$ Higgs fields to break the EW and $U(1)^{\prime}$ gauge symmetries. In addition, there are two $S O(10)$ adjoint Higgs fields, $\mathbf{4 5}_{H}$ and $\mathbf{4 5}_{H}^{\prime}$, in our model. They develop nonzero VEVs, and then the $S O(10)$ gauge symmetry breaks down to $G_{S M} \times U(1)^{\prime}$ at the GUT scale. The low-energy effective theory is the $U(1)^{\prime}$ extension of the SUSY SM with the 10- and 16-dimensional matter fields.

After the $U(1)^{\prime}$ symmetry breaking, the chiral superfields $\hat{D}_{R i}^{c}$ and $D_{R i}^{\prime c}\left(\hat{L}_{L i}\right.$ and $\left.L_{L i}^{\prime}\right)$ mix each other, and we find the massless modes that correspond to the SM right-handed down-type quarks and left-handed leptons. Eventually, the relevant Yukawa couplings for quarks and charged leptons are described as

$$
W_{Y}^{S S M}=h_{u i j} Q_{L i} U_{R j}^{c} H_{u}+Y_{d i j} Q_{L i} D_{R j}^{c} H_{d}+Y_{e i j} L_{L i} E_{R j}^{c} H_{d}+\widetilde{\mu}_{i j}\left(\overline{D_{R h i}^{c}} D_{R h j}^{c}+\overline{L_{L h i}} L_{L h j}\right) .
$$


$D_{R i}^{c}$ and $L_{L i}\left(D_{R h i}^{c}\right.$ and $\left.L_{L h i}\right)$ are the (heavy) chiral superfields of right-handed down-type quarks and left-handed leptons in the mass bases. The Yukawa coupling constants for the SM down-type quarks and charged leptons are described as

$$
\left(Y_{d}\right)_{i j}=\left(h_{u i k}+\varepsilon_{d i k}\right)\left(\hat{U}_{D_{R}^{c}}\right)_{k j},\left(Y_{e}\right)_{i j}=\left(\hat{U}_{L_{L}}^{T}\right)_{i k}\left(h_{u k j}+\varepsilon_{e k j}\right),
$$

using the mixing parameters, $\left(\hat{U}_{D_{R}^{c}}\right)_{k j}$ and $\left(\hat{U}_{L_{L}}\right)_{i k}$, and the additional parameters, $\varepsilon_{d, e k j}$, originated from the higher dimensional parameters. Then, the realistic Yukawa couplings are realized by the mixing between 10- and 16-dimensional matter fields.

Since the fields in $\mathbf{1 0}$ and $\mathbf{1 6}$ representations carry different $U(1)^{\prime}$ charges, the SM fields have flavor-dependent $U(1)^{\prime}$ interaction $[2,3]$. The $U(1)^{\prime}$ gauge interaction of right-handed downtype quarks and left-handed leptons is described in the interaction basis as $\mathscr{L}_{g}=-i g_{X}\left(3 \overline{\hat{\varphi}}_{i} \not^{\prime} \hat{\varphi}_{i}-\right.$ $\left.2 \overline{\varphi^{\prime}}{ }_{i} Z^{\prime} \varphi_{i}^{\prime}\right)$, where the factors 3 and -2 are $U(1)^{\prime}$ charges for the fermionic components $\hat{\varphi}_{i}$ and $\varphi_{i}^{\prime}$ of the chiral superfields $\hat{\psi}_{i}$ and $\psi_{i}^{\prime}$. $Z^{\prime}$ is the $U(1)^{\prime}$ gauge boson and $g_{X}$ is defined as $g_{X}=g / \sqrt{40}$ at GUT scale, where $g$ is the $S O(10)$ gauge coupling constant. Using the unitary matrix $U_{\psi}$, we define the flavor-violating couplings $A_{i j}^{\varphi}$ for the SM fermions as $\mathscr{L}_{g}=-i g_{X} \bar{\varphi}_{i}\left(5\left(\hat{U}_{\psi}^{\dagger} \hat{U}_{\psi}\right)_{i j}-2 \delta_{i j}\right) \not Z^{\prime} \varphi_{j} \equiv$ $-i g_{X} A_{i j}^{\varphi} \bar{\varphi}_{i} \not{ }^{\prime} \varphi_{j}$, where $\varphi$ is the fermion component of the chiral superfield $\psi$ in the mass base and denotes right-handed down-type quark $\left(d_{R}^{c}\right)$ and left-handed lepton $\left(l_{L}\right)$. As discussed in Refs. $[2,3]$, we find that all elements of the flavor violating couplings are $O(1)$, so that we need careful analyses of their contributions to flavor physics, even if the $Z^{\prime}$ boson is quite heavy.

\subsection{Flavor physics}

The large tree-level FCNCs involving the $Z^{\prime}$ boson are promised in our model. Here, we sketch the relevant constraints on the flavor-violating $Z^{\prime}$ interactions and give future prospects. The detail of the analysis is shown in Ref. [3].

In our model, the SUSY SM Higgs doublets are charged under $U(1)^{\prime}$, so that their nonzero VEVs contribute to the $Z^{\prime}$ mass $\left(m_{Z^{\prime}}\right)$ as well as the SM gauge bosons. The mass mixing between $Z$ and $Z^{\prime}$ is generated by the VEVs as well. Then the $Z^{\prime}$ interaction is described by the mixing and the tree-level FCNCs. The flavor-violating couplings significantly contribute to $B_{d / s}^{0}-\bar{B}_{d / s}^{0}$ and $K^{0}-\bar{K}^{0}$ mixings, flavor-violating decays, and $\mu-e$ conversion in nuclei. In particular, the CP violation in the $K^{0}-\bar{K}^{0}$ mixing gives the stringent constraint on the $Z^{\prime}$ scale [3]. In the lepton flavor violation (LFV), the most relevant processes would be the $\mu \rightarrow 3 e$ process and the $\mu$-e conversion process.

The LFV $\mu$ decay has been investigated at the SINDRUM experiment: $\operatorname{BR}(\mu \rightarrow 3 e)<1.0 \times$ $10^{-12}$ [9]. The coming experiment will reach $\mathscr{O}\left(10^{-16}\right)$ [10]. The left panel of Fig. 1 shows the correlation between $\delta\left(\varepsilon_{K}\right)$ and $\operatorname{BR}(\mu \rightarrow 3 e)$, setting and $\Lambda_{Z^{\prime}}=500 \mathrm{TeV}^{*} \delta\left(\varepsilon_{K}\right)$ describes the deviation of $\varepsilon_{K}$ from the SM prediction. The green region is excluded by the SINDRUM experiment [9] and the dashed green line corresponds to the expected upper bound in the Mu3e experiment [10]. According to the figures, we can expect that $\operatorname{BR}(\mu \rightarrow 3 e)$ is less than $\mathscr{O}\left(10^{-15}\right)$, as far as $\Lambda_{Z^{\prime}}>500 \mathrm{TeV}$. When $\Lambda_{Z^{\prime}}$ is $500 \mathrm{TeV}$ which correspond to $m_{Z^{\prime}} \simeq 36 \mathrm{TeV}, \mathrm{BR}(\mu \rightarrow 3 e)$ is about $3.5 \times 10^{-16}$ and can exceed the future sensitivity.

The right panel of Fig. 1 shows the correlations on $\delta \varepsilon_{K}$ and the $\mu$-e conversions. The dashed green lines are the future prospects for $\mathrm{BR}(\mu \mathrm{Al} \rightarrow e \mathrm{Al})$ of the COMET-II experiment $[11,12]$.

\footnotetext{
${ }^{*} \Lambda_{Z^{\prime}}$ corresponds to $m_{Z^{\prime}} / g_{X}$ approximately. The explicit definition of $\Lambda_{Z^{\prime}}$ is given in Ref. [3].
} 

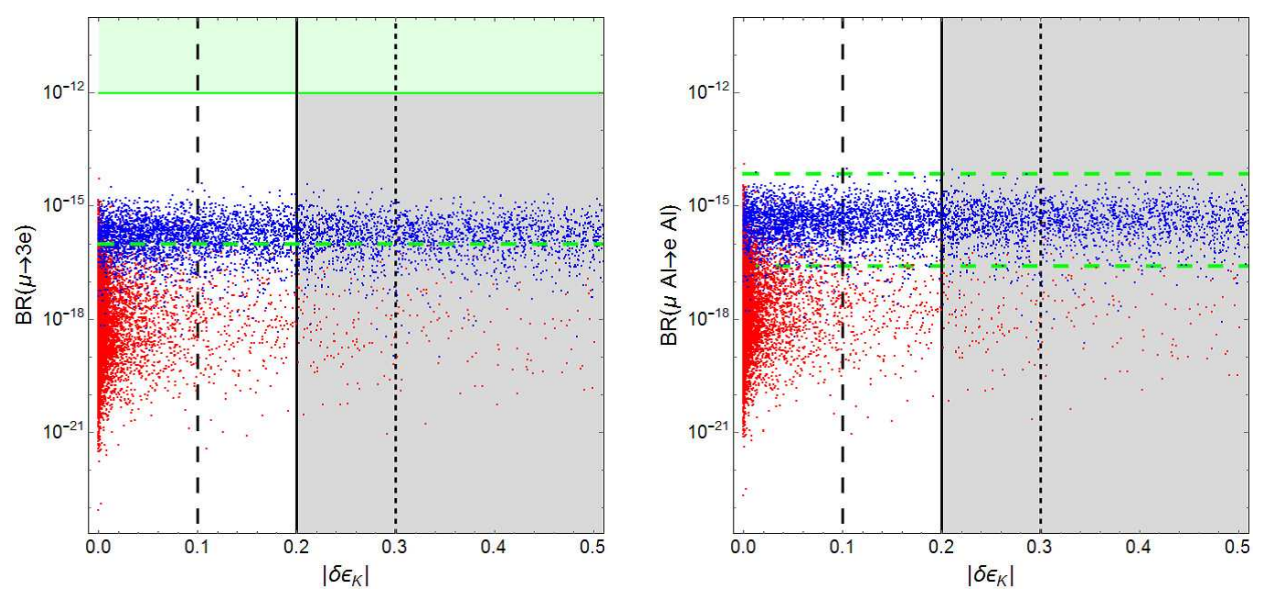

Figure 1: Our predictions for $\mathrm{BR}(\mu \rightarrow 3 e)$ (left panels) and $\mathrm{BR}(\mu \mathrm{Al} \rightarrow e \mathrm{Al})$ (right panels). We set $\Lambda_{Z^{\prime}}=$ $500 \mathrm{TeV}$ in both panels. The coefficients of higher-dimensional operators satisfy $\left|\varepsilon c_{i j}^{d}\right|<10^{-2}$ (red) and $\left|\varepsilon c_{i j}^{d}\right|<10^{-3}$ (blue). In the left panels, green region shows the experimental bound [9]. In the right panels, two green dashed lines show future sensitivity from COMET-I (upper one) and COMET-II (lower one) experiment $[11,12]$.

There is a chance to reach the future sensitivity in the mode of $\mu \mathrm{Al} \rightarrow e \mathrm{Al}: \mathrm{BR}(\mu \mathrm{Al} \rightarrow e \mathrm{Al}) \simeq$ $10^{-15}$ when $\Lambda_{Z^{\prime}}$ is set to $500 \mathrm{TeV}$.

\section{Study of DM model}

Next, we discuss an extended SM, motivated by DM. From the phenomenological point of view, one simple setup is as follows.

We introduce an extra down-type quark, $F$, carrying SM charges as in Table 2. $F$ is a Dirac

\begin{tabular}{cccccc}
\hline \hline Fields & spin & $\mathrm{SU}(3)_{c}$ & $\mathrm{SU}(2)_{L}$ & $\mathrm{U}(1)_{Y}$ & $\mathrm{U}(1)_{X}$ \\
\hline$F$ & $1 / 2$ & $\mathbf{3}$ & $\mathbf{1}$ & $-1 / 3$ & 1 \\
$X$ & 0 & $\mathbf{1}$ & $\mathbf{1}$ & 0 & -1 \\
\hline \hline
\end{tabular}

Table 2: Extra fields in our model with global $\mathrm{U}(1)_{X}$.

fermion and the charge assignment is the same as the one of the right-handed down-type quarks, $d_{R}^{i}$ $(i=1,2,3)$. In our notation, $\left(d^{1}, d^{2}, d^{3}\right)$ correspond to $(d, s, b)$. We assign a global $\mathrm{U}(1)_{X}$ charge to $F$ to distinguish it from the SM down-type quarks. In addition, we introduce a complex scalar, denoted by $X$, which is also charged under the $\mathrm{U}(1)_{X}$ symmetry. $X$ is stable thanks to the $\mathrm{U}(1)_{X}$ symmetry and is a DM candidate in our model. ${ }^{\dagger}$ The charge assignment is summarized in Table 2.

Now we can write down the potential for the extra quark and the scalar:

$$
\begin{aligned}
V= & m_{F} \overline{F_{L}} F_{R}+\lambda_{i} \overline{F_{L}} X^{\dagger} d_{R}^{i}+h . c . \\
& +m_{X}^{2}|X|^{2}+\lambda_{H}|X|^{2}|H|^{2}+\lambda_{X}|X|^{4}-m_{H}^{2}|H|^{2}+\lambda|H|^{4} .
\end{aligned}
$$

${ }^{\dagger}$ This setup could be derived from the GUT [6]. Recently, the explanation of the origin of the CKM matrix is also given in this kind of model [8]. 
Each of the Yukawa couplings, $\lambda_{i}$, induces the decay of $F: F \rightarrow X d^{i}$. Note that $V_{\mathrm{X}}$ includes the coupling of $X$ to the SM Higgs boson $(H)$. $\lambda_{H}$ plays a crucial role in dark matter physics, as discussed in Ref. [7].

In our model, there are several parameters that can be determined by combining the analyses of dark matter physics, flavor physics and the direct searches at the LHC. The relevant parameters in our study are as follows:

$$
m_{X}, m_{F}, \lambda_{H}, \lambda_{b}, \operatorname{Re}\left(\lambda_{s}\right), \operatorname{Im}\left(\lambda_{s}\right), \operatorname{Re}\left(\lambda_{d}\right), \operatorname{Im}\left(\lambda_{d}\right)
$$

Note that we can define $\lambda_{b}$ as a real one, without loss of generality. In order to avoid the stringent bounds from flavor physics and direct detections of DM, we assume the following relation,

$$
\left|\lambda_{b}\right| \gg\left|\lambda_{d}\right|,\left|\lambda_{s}\right|
$$

In this case, $F$ mainly decays to $X$ and the bottom quark, and the dominant annihilation process of $X$ is $X X^{\dagger} \rightarrow \bar{b} b$ through the $t$-channel exchange of $F$, as far as $\lambda_{H}$ is relatively small. This means that $\lambda_{b}$, as well as $m_{X}$ and $m_{F}$, can be fixed by the direct search for $F$ and $X$ in the $b \bar{b}$ signal accompanied by the large missing energy at the LHC and the DM observables, i.e., the relic abundance and the direct/indirect detections.

On the other hand, $\lambda_{d}$ and $\lambda_{s}$ are tiny in our setup, but not vanishing in general. The Yukawa couplings are strongly constrained by flavor physics and should be less than $\mathscr{O}(0.01)$, as we see in Sec. 2.1. In other words, we can expect the sizable deviations in physical observables in flavor violating processes. In fact, we will find some correlations among the observables in the $\Delta F=2$ processes and derive explicit predictions for them, taking the analyses of the DM and LHC physics into account.

\subsection{Flavor Physics}

The current status of this model at the LHC and the DM direct detection experiment is summarized in Refs. [7, 13]. In this paper, we mainly discuss flavor physics in this DM model. In our model, FCNCs are induced by the Yukawa couplings between quarks and the dark matter at the one-loop level. Since the chiralities of quarks are right-handed in the Yukawa coupling, we find that the new physics contributions to the flavor violating processes are strongly suppressed. In the massless limit of the SM quarks, the box diagrams involving $X$ and $F$, induce the operators relevant to the $\Delta F=2$ processes [7]:

$$
\mathscr{H}_{\text {eff }}^{\Delta F=2}=\left(\widetilde{C}_{1}\right)_{i j}\left(\overline{d_{R}^{i}} \gamma^{\mu} d_{R}^{j}\right)\left(\overline{d_{R}^{i}} \gamma^{\mu} d_{R}^{j}\right)+h . c . .
$$

The $K_{0}-\overline{K_{0}}, B_{d}-\overline{B_{d}}$, and $B_{s}-\overline{B_{s}}$ mixing are well investigated theoretically and experimentally. Since $\lambda_{b}$ is $\mathscr{O}(1)$ [7], $B_{d}-\overline{B_{d}}$ and $B_{s}-\overline{B_{s}}$ mixing become important even if $\left|\lambda_{d}\right|$ and $\left|\lambda_{s}\right|$ are small compared to $\lambda_{b}$. Besides, the physical observables associated with $K_{0}-\overline{K_{0}}$, in general, constrain new physics contributions, although their SM predictions still have large uncertainties.

In Ref. [7], we have investigated our predictions in the following observables:

$$
\Delta M_{B_{d}}, \Delta M_{B_{s}}, S_{\psi K}, S_{\psi \phi}, \varepsilon_{K}
$$



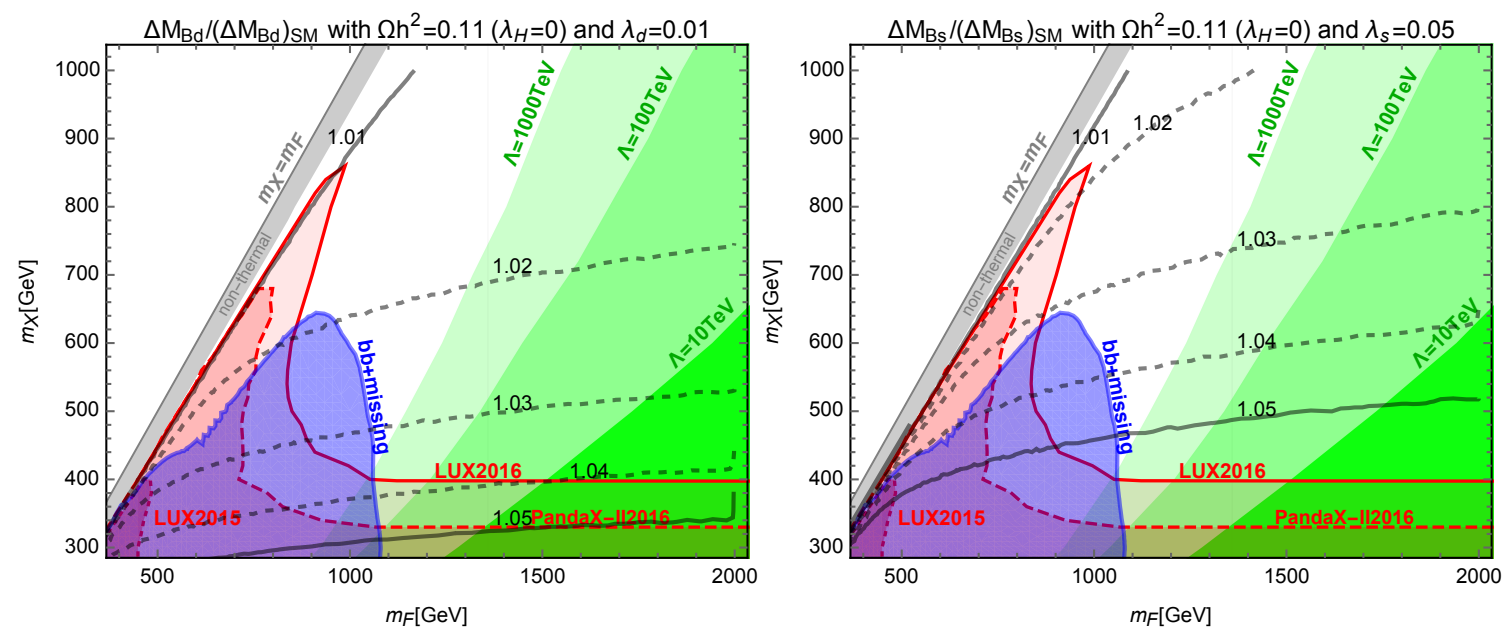

Figure 2: Predictions for the $\Delta F=2$ processes. $\Omega h^{2}=0.1198 \pm 0.0015$ [5] is satisfied. The blue region is excluded by the $b b+E_{T}^{\text {miss }}$ at the LHC [7, 13]. The red region is excluded by the LUX experiment [14]. In the green region, the Landau poles appear below $1000 \mathrm{TeV}$.

$\Delta M_{K}$ is not included, because of the large theoretical ambiguity. Among our parameters summarized in Eq. (2.2), we expect that $m_{F}, m_{X}$ and $\lambda_{b}$ are determined by the observables in the DM physics and the LHC experiments. Then, the other parameters, $\operatorname{Re}\left(\lambda_{s}\right), \operatorname{Im}\left(\lambda_{s}\right), \operatorname{Re}\left(\lambda_{d}\right)$ and $\operatorname{Im}\left(\lambda_{d}\right)$, are fixed by the observables in Eq. (2.5). The number of the parameters is smaller than the one of the observables, so that we can obtain an explicit prediction for the physical quantities measured by the flavor experiments.

In the $B_{d}-\overline{B_{d}}$ and $B_{s}-\overline{B_{s}}$ mixing, the representative observables relevant to the mixing are mass differences denoted by $\Delta M_{B_{d}}$ and $\Delta M_{B_{s}}$. They are influenced by $\left(\widetilde{C}_{1}\right)_{b d}$ and $\left(\widetilde{C}_{1}\right)_{b s}$. In Fig. 2, we can see the deviations of $\Delta M_{B_{d}}$ and $\Delta M_{B_{s}}$ from the SM predictions, fixing $\lambda_{d, s}$ at $\lambda_{d}=0.01$ (left panel) and $\lambda_{s}=0.05$ (right panel). $\lambda_{b}$ is fixed to satisfy the correct DM density. The regions excluded by the LHC (blue), the LUX experiments (red) and the triviality bound (green) are shown in the figures. The solid lines predict $1 \%$ and $5 \%$ deviations respectively, compared to the SM predictions. The dotted lines correspond to the $2 \%, 3 \%$, and $4 \%$ deviations from top to bottom in each panel. As we see in those figures, the deviations are enough small to evade the bounds on the $\Delta F=2$ processes, as far as $\left|\lambda_{d}\right| \leq 0.01$ and $\left|\lambda_{s}\right| \leq 0.05$ are satisfied. Note that there are still large uncertainties of the SM predictions for $\Delta M_{M_{q}}$. If $\lambda_{d}\left(\lambda_{s}\right)$ is set to $0.02(0.1)$, the deviations become about four times bigger than the values on Fig. 2. Then, we could conclude that the upper bounds on $\left|\lambda_{d}\right|$ and $\left|\lambda_{s}\right|$ are $\mathscr{O}(0.01)$ and $\mathscr{O}(0.05)$ respectively, in the region that the Landau poles do not appear below $1000 \mathrm{TeV}$ [7].

In Fig. 3, we can see the bounds on the $\Delta F=2$ processes, more clearly. We fix $m_{X}$, and $m_{F}$ and $\lambda_{b}$, according to the requirement of the correct relic density within $1 \sigma$. We choose the reference point: $\left(m_{X}, m_{F}, \lambda_{b}\right)=(900 \mathrm{GeV}, 964.4 \mathrm{GeV}, 0.66)$. On the blue bands, the deviations of $S_{\psi K}$ and $S_{\psi \phi}$ are within $1 \sigma: S_{\psi K}=0.691 \pm 0.017$ and $S_{\psi \phi}=0.015 \pm 0.035$. On the red (dashed) lines, the deviations of $\Delta M_{M_{q}}$ are $5 \%$ (-5\%). The pink (dashed) lines predict $10 \%$ (-10\%) deviations, respectively. In the red regions, the magnitudes of the deviations are less than $5 \%$. We see that the region where the magnitudes of the deviations are less than $5 \%$ for the $\Delta F=2$ processes 

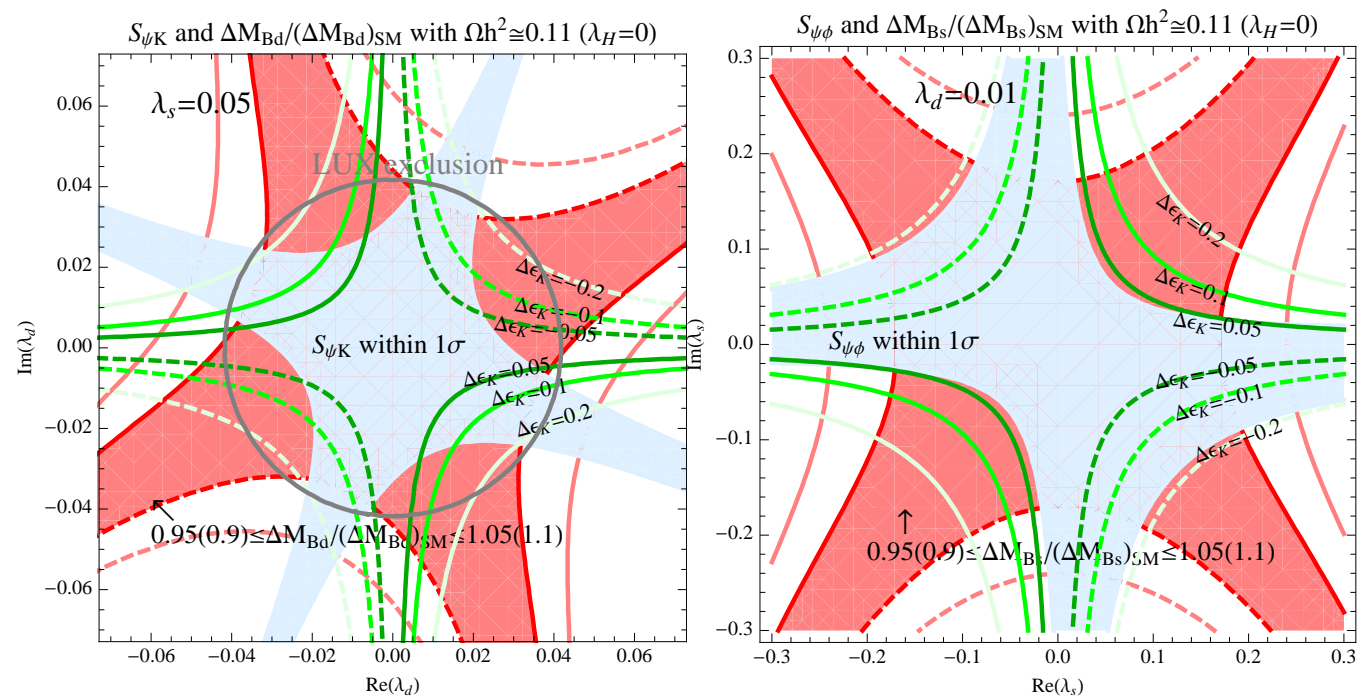

Figure 3: Predictions for the $\Delta F=2$ processes. The other parameters are fixed by the relic abundance within $1 \sigma:\left(m_{X}, m_{F}, \lambda_{b}\right)=(900 \mathrm{GeV}, 964.4 \mathrm{GeV}, 0.66)$. The gray circle depicts the exclusion of the LUX experiment [14]. The outside of the circle exceeds the upper bound on the cross section of the DM direct detection.

corresponds to $\left|\lambda_{d}\right| \lesssim 0.04$ (left panel) and $\left|\lambda_{s}\right| \lesssim 0.2$ (right panel) in Fig. 3 .

We can find the correlation between the flavor physics and the DM direct detection, especially in the left panel of Fig. 3. If $\left|\lambda_{d}\right|$ is sizable, the tree-level diagram, $X^{\dagger} d \rightarrow X^{\dagger} d$, induces significant deviations. The gray circle is the exclusion limit of the LUX experiment [14]. Then, we see that the allowed region roughly corresponds to $\left|\lambda_{d}\right| \lesssim 0.04$. In the right panel of Fig. 3, the exclusion limit is out of the figure.

The observables of the $K_{0}-\overline{K_{0}}$ mixing can be estimated. The physical observables on the $K_{0}-\overline{K_{0}}$ mixing are denoted by $\varepsilon_{K}$ and $\Delta M_{K}$. The predictions for the deviations of $\varepsilon_{K}$ are depicted as green lines in Fig. 3. Once the deviations of the $B_{d}-\overline{B_{d}}$ and $B_{s}-\overline{B_{s}}$ mixing are discovered, we can principally predict the deviation of $\varepsilon_{K}$. The (dashed) dark, normal and light green lines depict the (-)5\%, (-)10\%, and (-)20\% deviations of $\varepsilon_{K}$ respectively, compared to the SM prediction. On each panel, $\lambda_{s}=0.05$ (left) and $\lambda_{d}=0.01$ (right) are assumed. In Fig. 3, they correspond to $\left(\Delta M_{B_{s}} /\left(\Delta M_{B_{s}}\right)_{\mathrm{SM}}, S_{\psi \phi}\right)=(1.004,0.037)$ (left) and $\left(\Delta M_{B_{d}} /\left(\Delta M_{B_{d}}\right)_{\mathrm{SM}}, S_{\psi K}\right)=(1.003,0.687)$ (right). As we see in the left panel of Fig. $3,\left|\Delta \varepsilon_{K}\right|$ cannot exceed about 0.2, on this mass point.

In conclusion, we study flavor physics in two kinds of Beyond Standard Models. In both models, $\varepsilon_{K}$ gives a stringent constraint on the new physics scale. In the $\mathrm{SO}(10) \mathrm{GUT}$, we propose the LFV $\mu$ decays to prove the new physics. In the DM model, the DM relic abundance requires large Yukawa couplings so that we suggest that the $\Delta B=2$ processes are also important in testing the simple DM model. The future experiment [15] could also cover our parameter region [7].

The author would like to thank T. Abe, J. Hisano, J. Kawamura, Y. Muramatsu, S. Okawa, and M. Yamanaka for fruitful collaboration. 


\section{References}

[1] H. Georgi, AIP Conf. Proc. 23, 575 (1975); H. Fritzsch and P. Minkowski, Annals Phys. 93, 193 (1975).

[2] J. Hisano, Y. Muramatsu, Y. Omura and M. Yamanaka, Phys. Lett. B 744, 395 (2015) [arXiv:1503.06156 [hep-ph]].

[3] J. Hisano, Y. Muramatsu, Y. Omura and Y. Shigekami, JHEP 1611, 018 (2016) [arXiv:1607.05437 [hep-ph]].

[4] G. Hinshaw et al. [WMAP Collaboration], Astrophys. J. Suppl. 208:19 (2013) [arXiv:1212.5226 [astro-ph.CO]].

[5] P. A. R. Ade et al. [Planck Collaboration], Astron. Astrophys. 594, A13 (2016) [arXiv:1502.01589 [astro-ph.CO]].

[6] P. Ko, Y. Omura and C. Yu, JHEP 1604, 098 (2016) [arXiv:1601.00586 [hep-ph]].

[7] T. Abe, J. Kawamura, S. Okawa and Y. Omura, JHEP 1703, 058 (2017) [arXiv:1612.01643 [hep-ph]].

[8] S. Okawa and Y. Omura, arXiv:1703.08789 [hep-ph].

[9] U. Bellgardt et al. [SINDRUM Collaboration], Nucl. Phys. B 299 (1988) 1.

[10] A. Blondel, A. Bravar, M. Pohl, S. Bachmann, N. Berger, M. Kiehn, A. Schoning and D. Wiedner et al., arXiv:1301.6113 [physics.ins-det].

[11] Y. Kuno [COMET Collaboration], PTEP 2013, 022C01 (2013).

[12] COMET Collaboration, (Available at: http://comet.kek.jp/Documents_files/IPNS-Review-2014.pdf)

[13] J. Kawamura and Y. Omura, Phys. Rev. D 93, no. 11, 115011 (2016) [arXiv:1601.07396 [hep-ph]].

[14] D. S. Akerib et al., arXiv:1608.07648 [astro-ph.CO].

[15] E. Aprile et al. [XENON Collaboration], JCAP 1604, no. 04, 027 (2016) [arXiv:1512.07501 [physics.ins-det]]. 